\title{
Repeat use of contraceptive crisis services among adolescent women
}

\author{
Jane Meyrick, BA, PhD, Head of Standards and Review
}

Correspondence: Dr Jane Meyrick, Head of Standards and Review, Health Development Agency, Trevelyan House, 30 Great Peter Street, London, SW1P 2HW, UK. Tel: 0207222 5300, Fax: 02074138900.

(Accepted August $24^{\text {th }}, 2000$ )

\begin{abstract}
Summary
"They don't get pregnant twice unless they are hopeless."

This was one Doctor's reported ${ }^{l}$ assessment of women who had more than one abortion.

There is some evidence that the repeated use of pregnancy testing 'scares', emergency contraception and abortion is increasing across all women. However, there may also be an interaction between this general trend and the difficulties faced by particularly vulnerable groups of teenagers who also have higher rates of teenage parenthood. This paper aims to provide an overview of the research and international statistics in this sparsely researched area. It will draw on the author's own qualitative work with 'high risking' teenage girls, and that of other researchers, in order to attempt to reach an understanding of the mechanisms behind this increasingly common phenomenon. The indications from this work refutes the notion that these women form a special or 'hopeless' group, but point towards general problems with contraception and services common to all women that may become compounded through structural vulnerability such as deprivation.
\end{abstract}

\section{Key words}

crisis contraception, repeat abortion, teenage pregnancy

\footnotetext{
Key message points

- More research needs to be done around all presentations for crisis contraception, pregnancy testing, emergency contraception and termination.

- Teenagers do not have the highest termination of pregnancy rates.

- Repeated abortion is increasingly common among all women.

- Research indicates that repeat use of contraceptive crisis may be symptomatic of difficulties with contraception common to all women.
}

\section{Introduction}

Teenagers in general are poor contraceptive users and tend to delay accessing services once sexually active (up to 1 year), attendance generally being precipitated by a 'pregnancy scare'. 2,3 However, much of the research work into this aspect of sexual behaviour is outdated, based on American teenagers or on smaller, qualitative UK studies. This paper will try and draw from research around contraceptive crisis points (pregnancy testing and emergency contraception), and then terminations of pregnancy, in order to reveal the mechanisms behind the trends.

\section{Pregnancy scares and emergency contraception}

Zabin et $\mathrm{al}^{4}$ studied a sample $(\mathrm{n}=2929)$ of American women, aged 17 or under, requesting pregnancy tests from a variety of clinics. For $46.6 \%$ of these women, the index test was not the only pregnancy test they had ever had. Of those who conceived, one in three had previously had at least one negative pregnancy test before a positive one. Zabin used this research to show an unmet need for targeted prevention work with women on presentation to acute services for emergency contraception or pregnancy testing. More research needs to be done around all presentations for crisis contraception, pregnancy testing, emergency contraception and termination.

High level use of emergency contraception has been noted in the University of Durham in monitoring research. ${ }^{5}$

In a qualitative study of high contraceptive risking taking (termination or emergency contraception) amongst teenage women, ${ }^{6}$ repeated previous attendance at services for either contraception, emergency contraception and earlier terminations emerged as a common experience. Of a small sample, one young woman was attending for her third abortion, one for her second and a third reported having lost a previous pregnancy. Technical difficulties in using contraceptive methods (especially the pill and condom) lead to the need to access crisis contraceptive services rather than failure to use contraception. The relevance of ongoing problems in the use of contraception is supported in a similar study by Goraya and Prakash. ${ }^{7}$ A picture emerged of young women experiencing technical problems with contraception coupled with a poor relationship with available services that resulted in attendance only at the crisis point of a 'pregnancy scare'. Previous use of acute services such as earlier terminations had not been used to address their existing contraceptive difficulties. This is supported by the US work of $\mathrm{Zabin}^{4}$ and by findings of a qualitative study by the $\mathrm{HEA}^{8}$ in which one female from a deprived area reported of services:

"I wouldn't go back, they were horrible,....I went in there and it was really intimidating.... she was like 'well why have you come in here to get the morning after pill?' and I was like, 'the condom split', and she was,

'well shouldn't you have been more careful, be more careful next time'. I was, like, 'well it was an accident' and I felt really angry".

Of young women in this study $\left(\mathrm{HEA}^{8}\right)$ who had had a termination, most appeared to have expected some sort of counselling on being told they had an appointment for a 'consultation'. Instead they had been disappointed to find out that this is simply an appointment to arrange the termination itself, and that they were asked only cursory questions about their medical background. 
The emerging patterns of difficulty with both contraceptive use and services may be more problematic for vulnerable young people in relation to repeated contraceptive crisis. The $\mathrm{HEA}^{8}$ work found that many of those who were described as vulnerable (definition based on combination of sexually active, not using contraception and coming from a deprived area) demonstrated a 'defeatist' or 'fatalistic' attitude to contraception. This attitude seemed to parallel a broader difficulty in exercising control over their lives, and would indicate that experiencing contraceptive problems may result in more vulnerable teenagers simply abandoning attempts to use contraception altogether.

\section{Terminations of pregnancy \\ Age}

Data have shown that the percentage of conceptions terminated by abortion varies by age group. Overall trends since the mid 1970s are little changed in the proportion of abortions to the under 16 group (over half all underage pregnancies), but a rising proportion of abortions for under 20 s is apparent, currently at more than a third. ${ }^{9}$ Pregnant teenagers are also more likely to have a late abortion (over 20 weeks gestation) than women aged 20 or above. ${ }^{10,11}$ However, international data show that terminations are most common in married women and women with children. ${ }^{12}$

\section{Deprivation}

The proportion of pregnancies terminated is lower in areas of greater social deprivation and areas with less public welfare, whereas adolescents who terminate their pregnancies tend to come from a higher socio-economic background, have done well at school and have higher educational aspirations. ${ }^{13-16}$ Parenthood tends to be avoided by pregnant adolescents who have more concrete and attainable future plans. ${ }^{17}$

Moral objections to abortion were found to be common and even greater in sexually active females from deprived areas. ${ }^{8}$ Staying pregnant was often less of a conscious decision and more of an acceptance of what had happened, and was accompanied by a sense of fatalism. The research was clear that abortion was not regarded as a form of contraception. In general there seemed to be a marked reluctance to consider abortion, even though relationships with the father had ended. ${ }^{18}$

\section{International trends in repeat abortions}

Overall, adolescents do not obtain a disproportionate level of abortions compared to older women. The repeat abortion trends across countries, expressed as a percentage of all abortions, for 1985/86 in all women are summarised in

Table 1 The repeat abortion trends across countries expressed as a percentage of all abortions, for 1985/86 in all women. (Figure given for Canada relates to 1993 data).

\begin{tabular}{lc}
\hline Country & Percentage \\
\hline${ }^{*}$ US & $44 \%$ \\
${ }^{*}$ Denmark & $36 \%$ \\
*England and Wales & $27 \%$ \\
${ }^{*}$ France & $19 \%$ \\
${ }^{*}$ Hungary & $49 \%$ \\
${ }^{*}$ Netherlands & $22 \%$ \\
${ }^{*}$ Sweden & $35 \%$ \\
${ }^{+}$Canada & $29 \%$ \\
\hline
\end{tabular}

* Osler, David and Morgall ${ }^{19}$

+The repeat rate for 1993. Millar, Wadhera and Henshaw 20
Table 1. However, under-reporting of past abortions may mean that these rates are, in fact, higher. ${ }^{19}$

Reliance on abortion in the US has declined. ${ }^{21}$ A study of $(\mathrm{n}=2001)$ terminations carried out in Kansas between 1991 and $1992^{22}$ found that women having repeat terminations were younger at first pregnancy (average age 18.7 vs. 19.7 years), and also that $28 \%$ of the sample had had no post abortion counselling.

\section{Trends in the UK: all women}

More women in general are having repeat abortions. ${ }^{23}$ The $\mathrm{ONS}^{24}$ reports rates of repeated abortion and births as shown in Figure 1. These data on repeat abortions show a small increase across women of all ages. However, this increase is not found in the corresponding data on women having an abortion with a previous live birth.

Figure 1 Percentage of women having an abortion who have had a previous abortion (ONS, 1998) ${ }^{24}$

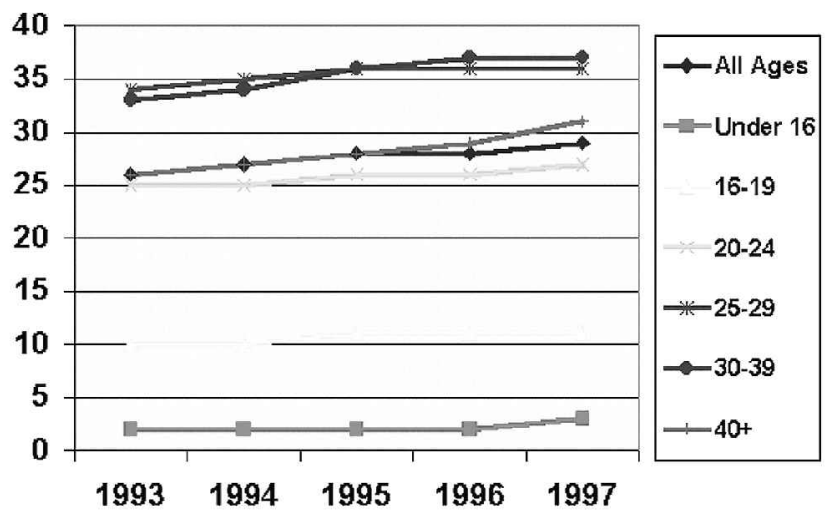

Higher rates amongst older women are also related to longer sexual 'careers' i.e. length of time they had been having sex. ${ }^{25}$ One US study describes the termination histories from a 1994/95 national survey of 9985 abortion patients. For $45 \%$ of the sample this was not their first abortion, and $7 \%$ reported three or more. However, UK data (Figure 1) show that the second abortion rate for 16-19 year olds has been steady at $11 \%$ since 1995 .

\section{Trends in the UK: younger women}

Only $1 \%$ of under 16 years olds in the UK have had a birth then an abortion, and 3\% have had a previous abortion.

Scottish data found that in $1997,38 \%$ of $16-19$ year old conceptions ended in an abortion. ${ }^{26}$ Of those aged 13-15 years, six were previously pregnant (one of whom gave birth). Of those aged 16-19 years, $24 \%(n=1076)$ were previously pregnant either once $(\mathrm{n}=880)$, twice $(\mathrm{n}=165)$ or three times $(\mathrm{n}=31)$, and $447(50.8 \%)$ of these pregnancies resulted in a delivery. 26

\section{Contraceptive use and counselling: research \\ All women}

The evidence from studies of older women and comparison of termination groups points to the relevance of continued problems with contraception.

A Scandinavian study ${ }^{27}$ followed up women $(n=2925)$ having a first abortion between 1987 and 1991. They concluded that:

'The relative risk of a repeat of abortion was $70 \%$ higher if the pregnancy at first abortion was associated with contraceptive failure compared to failure to use any contraception.' 
A further study by the authors comparing groups of first $(\mathrm{n}=50)$ and second $(\mathrm{n}=50)$ time terminations of women aged 15-44 years found that:

'Except for being somewhat older, women having a second abortion were more similar than dissimilar to women having their first induced abortion'.

This finding has been corroborated in similar research. ${ }^{28}$

Skjeldestad ${ }^{27}$ found that women having more than two abortions reported technical problems with contraception, feared side effects of the pill and used less effective methods or effective methods less efficiently. Many of this group refused to be interviewed. The study concluded that these women were 'unluckier' and this conclusion has been supported in previous research. ${ }^{29}$

Westfall and Kallail ${ }^{22}$ reported that:

'The incidence of repeat abortion is largely related to the risk of pregnancy inherent in coital frequency and contraceptive practice not to unusual or deviant personality characteristics'.

The common thread of erratic use of contraception even following an abortion is supported in research with younger women. Hewell and Andrews ${ }^{30}$ found that of a sample of teenagers aged 14-19 years who had had an abortion $(\mathrm{n}=29)$ or who had had a previous negative pregnancy test $(\mathrm{n}=35), 75 \%$ of both groups selected reliable contraception ( $90 \%$ effective when used correctly) after their visit. However, only $10.3 \%$ and $5.8 \%$, respectively, reported using reliable contraception at further follow-up.

Research by Cullen and Boyle ${ }^{31}$ and the HEA Contraceptive Education Service ${ }^{32}$ found that doctors may check contraceptive use through closed questions such as 'are you using contraception or not?'. However, this does not reveal actual use and women may interpret this as 'ever used' whereas doctors see it as 'effective' use. Furthermore, detailed questioning in Boyle's study revealed an alarming $70 \%$ rate of non or insufficient use.

\section{Young women}

Access to advice around decision making when women attend services for an abortion seems limited. Research into women's experience of abortion is lacking, forcing us to rely on out of date studies.

An old study by Mortons, Williams and Hindell ${ }^{33}$ interviewed 50 women having abortions (nine under 18 years and 17 between $18-24$ years, the remaining 24 over 25 years). The study specifically looked at the 'educational effect' of the abortion. Many had received 'lectures' from health professionals with whom they had come into contact in order to obtain a termination.

In an HEA study ${ }^{8}$ those who had had an abortion would have welcomed counselling for the emotional distress it had caused, but did not receive any. Good intentions to change contraceptive behaviour were common:

"I am certainly not going to get like this again"

but these did not seem to be translated into long-term changes:

"This has completely turned me off sex. But you forget.

I'll go on the pill when I've got the nerve to got to the doctor."

Most of the women interviewed thought they would probably go on the pill, despite often still worrying about possible harmful side effects.

Meyrick $^{6}$ found previous abortions not uncommon in high contraceptive risking teenager girls. One 17 year old reported:

"After my abortion, they gave me 1 month of the pill,

I never went back to get anymore and don't use anything'.
This would suggest a spiral relationship developing around contraceptive difficulties and poor relationships with services. ${ }^{6}$ Contraceptive problems were not addressed until a 'crisis' forced the young women back to a service at which problems were not addressed and may even have been compounded through judgmental treatment. ${ }^{6,28,34}$ One 20 year old woman who had miscarried at 17 reported:

"Coming into hospital with an infection, they didn't explain anything, were unhelpful,......I thought it was due to using the pill. I'd been getting sick and migraines, so kept changing it, so I was too scared to use the pill'.

Franz and Reardon ${ }^{35}$ report that adolescents having a termination were significantly more likely to:

- be dissatisfied with the choice of abortion

- have abortions later

- be dissatisfied with services at the time of the abortion

- feel forced by circumstances to have the abortion

- report being misinformed at the time of the abortion

- report greater psychological stress.

There is also some small scale evidence of mothers pressurising young women into terminations, ${ }^{7}$ but partners tended to be against abortion. ${ }^{8}$

\section{Conclusion}

More research needs to be done around all women's presentations for crisis contraception, pregnancy testing, emergency contraception and termination, and both young men and women's difficulties in using contraception, within the context of relationships. Basic tracking of repeat abortions relies only on self-report data; some suggest that medical note linkage should be introduced. ${ }^{36}$

It would also be useful to examine relationships with services in more detail, including the attitudes of service providers around the issue of adolescent sexual activity and women's problems with existing contraceptive technology.

Repeated abortion may be a symptom of continued difficulty with contraceptive use for a variety of reasons including situational, intrapsychic and social factors. ${ }^{37}$ These difficulties are not addressed during attendance at acute services and are compounded by the often judgmental treatment of services. $6,28,34$

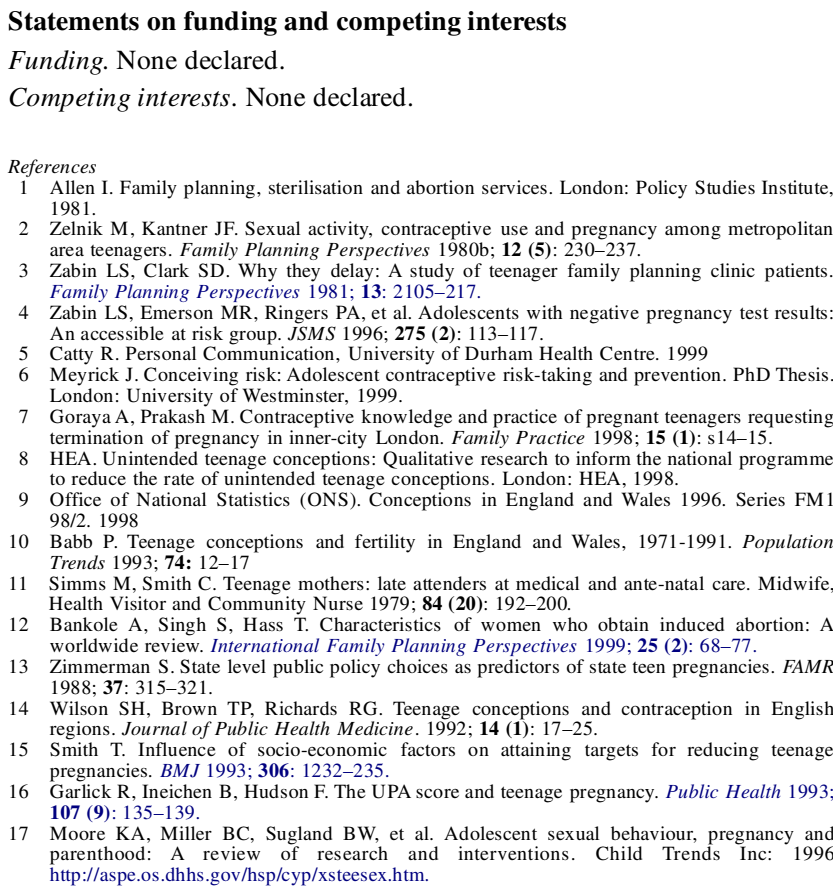


18 Tabberer S, Hall C, Webster A, et al. Teenage pregnancy and choice. Summary of interim

9 Osler M, David HP, Morgall JM. Multiple induced abortions: Danish experience. Patient Millar WJ, Wadhera S, Henshaw SK. Repeat abortions in Canada, 1975-1993. Family Planning Perspective 1997; 29 (1): 20-2

1 Alan Guttamacher Institute. Sex and America's Teenagers. New York: Alan Guttamacher Institute, 1994.

2 Westfall JM, Kallail KJ. Repeat abortion and use of primary care health services. Family Planning Perspectives 1995; 27: 162-165.

Botting B. Trends in Abortion. Population Trends 1991; 64: 19-29.

Office of National Statistics, Personal Communication. 1998

Henshaw SK, Kost K. Abortion patients in 1994-1955. Characteristics and contraceptive use. Family Planning Perspectives 1996; 28 (4): 140-158.

1983-1997. 1998

27 Skjeldestad FE. The incidence of repeat induced abortions - a prospective study. Acta Ostet Gyecol Scan 1994, 73: 706-710. 8 Houghton A. Women who have abortions: are they different? Journal of Public Health
29 Brower C. Three times unlucky: a study of women who have 3 or more legal abortions. $J$

30 Hewell SW, Andrews J L. Contraceptive use among female adolescents. Clinical Nursing Culn $D$. Wom (30): $356-363$.

20 reporting of patterns of contraceptive use prior to seeking abortion. Thesis submitted for Doctoral Degree in Clinical Psychology, supervised by Boyle, M. University of East London. 1998.

32 Contraceptive Education Service. Research Summary: Professionals, Contraception: Raising the issue. London: Health Education Authority, 1997.

Morton Williams J, Hindell K. Abortion and contraception: A study of patient's attitudes. London: Political and Economic Planning, 1972.

34 Pitts MK, Burtney E, Dobraszczyc U. There is no shame in it anymore: How providers of sexual health advice view young people's sexuality. Health Education Research 1996; 11 (1): $1-9$.

35 Franz W, Reardon D. Differential impact of abortion on adolescents and adults. Adolescence 1992; 27 (105): 161-172.

36 Gbolade GA. Record linkage analysis could have been used. BMJ 2000; 321: 381 (5 August). Tornbom M, Moller A. Repeat abortion: a qualitative study. J Psychosom Obstet Gynaeco 